\title{
Day Times Micromole per Liter per Kilogram per Meter Squared
}

National Cancer Institute

\section{Source}

National Cancer Institute. Day Times Micromole per Liter per Kilogram per Meter

Squared. NCI Thesaurus. Code C111173.

Days times micromoles per liter, divided by kilograms per meter squared. 